\title{
Assessment of possible airborne impact from risk sites: methodology for probabilistic atmospheric studies
}

\author{
A. A. Baklanov ${ }^{1}$ and A. G. Mahura ${ }^{1,2}$ \\ ${ }^{1}$ Danish Meteorological Institute, DMI, DK-2100, Copenhagen, Denmark \\ ${ }^{2}$ Institute of the Northern Environmental Problems, INEP, Kola Science Center, Apatity, 184200, Russia
}

Received: 21 July 2003 - Published in Atmos. Chem. Phys. Discuss.: 22 October 2003

Revised: 27 February 2004 - Accepted: 4 March 2004 - Published: 24 March 2004

\begin{abstract}
The main purpose of this study is to develop a methodology for a multidisciplinary nuclear risk and vulnerability assessment, and to test this methodology through estimation of a nuclear risk to population in the Northern European countries in case of a severe accident at the nuclear risk sites. For assessment of the probabilistic risk and vulnerability, a combination of social-geophysical factors and probabilities are considered.
\end{abstract}

The main focus of this paper is the description of methodology for evaluation of the atmospheric transport of radioactive releases from the risk site regions based on the long-term trajectory modeling. The suggested methodology is given from the probabilistic point of view. The main questions stated are: What are probabilities and times for radionuclide atmospheric transport to different neighbouring countries and territories in case of the hypothetical accidental release at the nuclear risk site? Which geographical territories or countries are at the highest risk from the hypothetical accidental releases?

To answer these questions we suggest applying the following research tools for probabilistic atmospheric studies. First tool is atmospheric modelling to calculate multiyear forward trajectories originated over the sites. Second tool is statistical analyses to explore temporal and spatial structure of calculated trajectories and evaluate different probabilistic impact indicators: atmospheric transport pathways, airflow, fast transport, typical transport time, maximum possible impact zone, maximum reaching distance, etc. These indicators are applicable for further GIS-analysis and integration to estimate regional risk and vulnerability in case of accidental releases at the risk sites and for planning the emergency response and preparedness systems.

Correspondence to: A. A. Baklanov

(alb@dmi.dk)

\section{Introduction}

The risks for airborne radioactive contamination and significant radiological consequences connected with sources in the Euro-Arctic region, in some cases predominantly affect the conditions at local and regional levels, yet in others appear to be far reaching, and of considerable concern for the whole region of concern. Thus, it is of particular interest to expound on issues such as:

- Which sources appear to be the main concern for those living close to and far from these sources?

- Which territories or countries are at the highest risk from the hypothetical accidental releases from nuclear risk sites?

For estimation of the potential nuclear risk and vulnerability levels, and for regional planning of radiological environmental monitoring networks and emergency preparedness systems, it is very important to determine for the nuclear risk sites (NRSs) of concern:

- geographical regions, territories, and/or countries most likely to be impacted;

- probabilities and times for various characteristics of atmospheric transport to geographical regions (including removal or precipitation factor) and their temporal and spatial variability;

- worst-case meteorological and accidental scenarios for detailed case studies;

- possible contamination and effects on population in case of an accident;

- site-sensitive hazards of potential airborne radioactive release; 
- vulnerability to a radioactive deposition concerning its persistence in the regional ecosystems:

- regional risks, socio-economical, and geographical consequences for different geographical areas and population groups.

Previously, several studies discussed possible approaches, and preliminarily investigated some of the mentioned important issues (e.g. see overview Baklanov, 2003). To study the possible consequences and risks from NRSs there could be two approaches - the case studies assessment (CSA) and the probabilistic risk assessment (PRA). The CSA is commonly used for estimation of possible doses for population. This approach is very useful to estimate possible consequences for typical or worst-case accidental scenarios and weather situations. However, it is computationally expensive for longterm (multiyear) simulation and further probabilistic assessments. Moreover, it is inconvenient for an analysis of factors of different nature (for example, geophysical processes of radionuclide transport and social-economical factors).

So, alongside with the first approach, for PRA some authors used simple dispersion models (mostly on a local scale). For example, the first map of risk due to severe accidents for majority of the European nuclear power plants (NPPs) showed the probability of excess cancer deaths (Slaper et al., 1994). The shortcoming of this approach was a limitation by a short distance, because the radioactive plume dispersion was evaluated by a simple Gaussian model using meteorological data only from one station. Andreev et al. (1998) simulated dispersion and deposition with a Lagrangian particle model and calculated the frequency of exceedance of certain thresholds for ${ }^{137} \mathrm{Cs}$ (regarded as a risk indicator). Additionally, GIS-based export/import matrices of risk were calculated for the European countries. Shortcoming of this method is the use of a limited number of meteorological situations, which can not satisfactory represent a long-term statistics.

Therefore, for PRA on a regional level some authors suggested to use more economical trajectory modelling approach. It should be noted that this approach was used for estimation of impacts from various air pollutants as shown by Eliassen and Saltbones (1983); other type of the probabilistic approach, based on the ensemble methods, was realized by Galmarini et al. (2001) for nuclear emergency preparedness. For the nuclear risk sources, for example, Mahura et al. (1999), Baklanov et al. (2002b) used an isentropic trajectory model and cluster analysis technique to assess possible impacts of the hypothetical nuclear accidents in the northern regions. In particular, the long-term consequences for the Nordic countries population were estimated on a basis of the Chernobyl accident exposures in Scandinavia (Moberg, 1991; Dahlgaard, 1994). Mapping of the regional nuclear risk and vulnerability was realized for Scandinavia based on integration of mathematical modelling and GIS-analysis (Rigina and Baklanov, 2002). Saltbones et al. (2000) also performed the long-term trajectory modelling and evaluated a few case studies of the long-range transport from the Kola NPP. However, they used 2-D trajectories, and risk mapping was not realized.

The studies of possible regional risk from the North-West Russian NRSs were initiated in 1991 in bounds of the "Risk" Project of the Russian State Programme "Ecological Safety of Russia" and projects in cooperation with the Kola NPP (Baklanov et al., 1992, 1994). These studies were continued during 1995-1997, and extended for NRSs of the Barents region in a series of international pilot studies/projects where the dispersion modelling, system analysis, and ranging of possible risk from different sites were employed (IIASA, 1996; Bergman and Baklanov, 1998; Bergman et al., 1998). During 1996-1997, another study was performed for the Bilibino NPP using trajectory modelling and cluster analysis to evaluate atmospheric transport pathways from NPP to the State of Alaska (Jaffe et al., 1997a; Mahura, 1998). During 1997-1998, a similar study which included more detailed probabilistic and several specific cases analyses, was realized for the Kola NPP (Jaffe et al., 1997b; Baklanov et al., 2001). During 1998-2000, for multidisciplinary analysis of nuclear risk in the Barents region the "Risk and Nuclear Waste" Programme was initiated (Baklanov and Bergman, 1999; ÖCB, 2000; Baklanov et al., 2001; Mahura et al., 2001). At the same period, the INTAS Project supplemented the ÖCB Project (Bergman, 1999; INTAS, 2000). Moreover, a joint study of the DMI and Novosibirsk Computing Centre of Russia suggested an alternative method for estimation of nuclear risk and vulnerability, based on the sensitivity theory and inverse modelling (Penenko and Baklanov, 2001).

So, it still remains an important issue to develop a methodology for the multidisciplinary nuclear risk and vulnerability assessments. Therefore, the on-going project of the Nordic countries "Atmospheric Transport Pathways, Vulnerability, and Possible Accidental Consequences from the Nuclear Risk Sites in the European Arctic" (AR-NARP, 2001-2003) is an extension and integration of previous studies in this field, and hence, the methodology (Baklanov and Mahura, 2001; Mahura and Baklanov, 2002; Baklanov et al., 2002b; Baklanov et al., 2003), which is elaborating in bounds of this project, is a logical continuation of our mentioned work.

\section{Methodology}

As it was mentioned, each of the two approaches - the probabilistic risk assessment and the case studies assessment - has some possibilities and shortcomings. Neither one of them is sufficient for the complex risk assessments. Therefore, as optimal, the following strategy for multidisciplinary methodology integrating different specific approaches is suggested:

- PRA approach for probabilistic atmospheric studies;

- combination of the PRA and CSA approaches; 
- radio-ecological sensitivity and specific nutrition pathways for studied regions;

- probabilistic safety assessments and probabilities of different severity accidents;

- possible social and economical loss and consequences;

- GIS-integration of different factors, probabilities, and indicators for risk and vulnerability mapping.

\subsection{General scheme for probabilistic risk assessment}

General scheme for the suggested methodology of the probabilistic risk assessments based on the long-term modelling is shown in Fig. 1. For assessment the following items should be considered:

1) social-geophysical factors:

- accident severity and release characteristics;

- proximity to risk sites and level of plume dispersion;

- population density in area;

- presence of critical groups of population;

- radio-ecological vulnerability of area;

- risk perception, preparedness of safety measures, emergency preparedness systems;

- economical and technical means, counteracting consequences of accident;

2) probabilities:

- probability of an accident of certain severity at the sites;

- probability of atmospheric transport from the sites towards regions of interest;

- probability of radionuclide removal over regions during atmospheric transport from the sites.

The following approaches and modelling tools are used in the suggested methodology for PRA studies:

- Trajectory modelling (e.g. 3-D isentropic trajectory model; Merrill et al., 1985) and 3-D DMI trajectory model (Sørensen et al., 1994)) to calculate multiyear forward trajectories originated over the NRS regions at various altitudes;

- Cluster analysis (Mahura, 1998; Mahura et al., 1999; Baklanov et al., 2002a) to identify atmospheric transport pathways from NRSs;
- Probability fields analysis (Mahura, 2001; Baklanov et al., 2002a) to construct monthly, seasonal, and annual probability fields for airflow, fast transport, precipitation factor, and other indicators to identify the most impacted geographical regions;

- Long-range transport modelling (e.g. DERMA; Sørensen, 1998; Baklanov and Sørensen, 2001) and DMI-HIRLAM (Sass et al., 2000) models) to simulate radionuclide transport and deposition for hypothetical accidental releases at NRSs, and to compare with results of trajectory modelling;

- Specific case studies (Bergman et al., 1998; Baklanov et al., 2001, 2002a) to estimate consequences for environment and population after hypothetical accidents using experimental models based on the Chernobyl effects for the Nordic countries (Moberg, 1991; Galmarini et al., 1992; Dahlgaard, 1994; Nielsen, 1998);

- Radioecological vulnerability to radioactive deposition (Bergman and Ågren, 1999; ØCB, 2000) to evaluate vulnerability concerning its persistence in the ecosystems with focus on transfer of certain radionuclides into food chains of key importance for the intake and exposure in a whole population and certain groups;

- Risk evaluation and mapping (Rigina, 2001; Rigina and Baklanov, 2002) to analyze socio-economical consequences for different geographical areas and various population groups taking into account socialgeophysical factors and probabilities and using geographic, demographic, administrative and economical databases in combination with the GIS-analysis.

The main focus of this paper is to describe the aspects of the suggested methodology for the probabilistic atmospheric studies based on the trajectory modelling approach for further risk and vulnerability analysis, namely the four upper blocks of Fig. 1. Here we do not consider aspects of the probabilistic dispersion and deposition modelling, which is a topic of a separate paper. Regarding to the fifth block shown in Fig. 1, the used GIS methods are discussed by Rigina (2001), Rigina and Baklanov (2002). It is devoted to problems of residential radiation risk and territorial vulnerability mapping with respect to NRSs. Their study suggested two methods, based on an integration of the mathematical modelling and GIS-based spatial analyses which allow merging together the separate aspects: probabilistic analysis, dose estimation, modelling of consequences, etc.

\subsection{Trajectory modelling approach}

In general, each computed atmospheric trajectory represents a pathway of an air parcel motion in time and space. There are a few approaches to model atmospheric trajectories. Two 


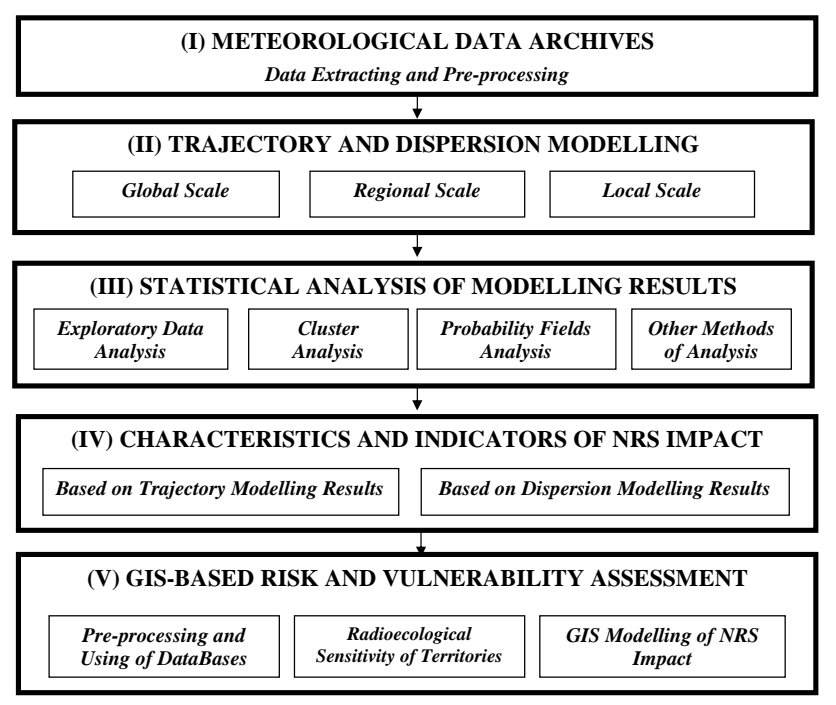

Fig. 1. Proposed scheme of the probabilistic risk assessment for atmospheric studies.

of these approaches are commonly used: isobaric and isentropic. The modelling of more realistic trajectories - "fully 3-D trajectories" - is preferable, although it is complex and it requires incorporation into simulation of large number of variables and parameters, and moreover, for the long-term statistics it increases significantly a computational time. In this study the isentropic approach was selected. Although this type of trajectory modelling uses assumption of adiabatically moving air parcels and neglects various physical effects, it is still a useful research tool for evaluating common airflow patterns within meteorological systems on various scales. Some uncertainties in these models are related to the interpolation of meteorological data, which might be sparsely measured, applicability of the considered horizontal and vertical scales, assumptions of vertical transport, etc. (Merrill et al., 1985; Kahl, 1996; Stohl, 1998).

As input data, a gridded dataset - NCEP Global Tropospheric Analyses - available at the NCAR archives was used. The original gridded wind fields were interpolated to potential temperature (isentropic) surfaces. It should be noted that quality of trajectory calculation is highly dependent on the original quality of the NCEP fields $\left(2.5^{\circ} \times 2.5^{\circ}\right.$ latitude vs. longitude), and it may not reflect the contribution of the frontal passages and local terrain phenomena. However, the trajectory errors rising during a single calculation might be smoothed in further analysis due to the large number of trajectories in the multiyear dataset. Among the higherresolution meteorological data, the DMI-HIRLAM (Sass et al., 2000) and ECMWF archives which were used in 3-D trajectory model (Sørensen et al., 1994) should be mentioned.

An interpolation procedure was performed for a multiyear period (1991-1996) applying a technique described by Merrill et al. (1985). Then, interpolated wind fields were used to compute forward trajectories from the site regions at standard UTC times at different potential temperature levels. These levels ranged from $255^{\circ} \mathrm{K}$ to $330^{\circ} \mathrm{K}$ with a step of $5^{\circ} \mathrm{K}$. In general, less than two percent of trajectories were missing because of absence of archived meteorological data.

Instead of calculating only one trajectory, four trajectories for every calculation were used. The initial points of trajectories are located at each corner of a $1^{\circ} \times 1^{\circ}$ of latitude vs. longitude box, where the site is in the centre of the box. Calculation of four trajectories simultaneously allowed evaluating a consistency of the wind field in the direction of the atmospheric transport. Although all calculated trajectories were used for further analysis, it should be noted that there are differences in the representation of the general flow along trajectories. The flow is considered to be a reasonably consistent along the transport pathway if all four trajectories had shown a similar direction (reflecting convergence of flow) of transport for one time period. Trajectories, showing a strong divergence of flow, are assigned to a category of the "complex trajectories". These trajectories reflect more uncertainties in the air parcel motion. These differences are not so important in evaluation of the general climatological patterns, but they can be significant in, for example, identification of source regions for air pollutants, evaluation of the nature of the specific events with recorded elevated concentration of species, tracking tracers in the atmosphere, etc.

For all NRSs, the most probable release heights would be within the boundary layer, i.e. within several hundred meters above the ground. Therefore, at the next step, from all trajectories only those trajectories originating at altitudes not more than $500 \mathrm{~m}$ were selected. The chosen trajectories for further statistical analysis are limited by duration of 5 days. This limitation was decided to use because of 1) quality and accuracy of trajectory calculations after 5 days drops significantly, 2) observing development frames of the synoptic scales systems in the Euro-Arctic region, as well as 3) relative proximity of the analyzed geographical regions from the sites of interest. Moreover, to study altitudinal variations in the flow patterns (in particular, within the boundary layer and free troposphere), trajectories originated over the site regions at altitudes of 1.5 and $3 \mathrm{~km}$ above sea level (asl) could be also considered.

\subsection{Statistical analysis approach}

\subsubsection{Cluster analysis for atmospheric transport pathways}

The cluster analysis is a variety of multivariate statistical analysis techniques, which could be used to explore the existing structure within data sets. The specific purpose of this analysis is to divide a data set into groups (or clusters) of similar variables (or cases). Miller (1981) initiated application of the cluster analysis on trajectories. The important output of his study was evaluation of the airflow climatology, in particular, over the long time periods. Then later, cluster analysis 
techniques on trajectories were used extensively by various researchers in different scientific fields.

In general, output of cluster analysis on trajectories can provide insights in the tracers transport, common atmospheric flow patterns for the sites of interest, identification of the source regions for atmospheric pollutants, etc. The cluster analysis is used to divide calculated trajectories into groups, which represent the major airflow transport regimes. The following criteria are used: latitude and longitude values at each time interval of trajectory. These represent both direction and velocity of air parcel motion. Similarity among trajectories in each cluster is maximized considering the full length of each forward trajectory. Within each cluster, individual trajectories can be averaged to obtain the mean cluster trajectory (or atmospheric transport pathway). Thus, the original large data set of trajectories can be reduced to a small number of mean cluster plots. These plots then can be interpreted, based on common synoptic conditions and features, and hence, the airflow climatology for the site can be summarized.

\subsubsection{Probability fields analysis for probabilistic impact in-} dicators

Probabilistic analysis is one of the ways to estimate the likelihood of occurrence of one or more phenomena or events. For each site a large number of forward trajectories that passed over various geographical regions was calculated. Each calculated trajectory contains information about longitude, latitude, altitude, pressure, temperature, relative humidity, etc. at each modelling time interval (in this study $-12 \mathrm{~h}$ ). The probability fields for these mentioned characteristics, either individual or combined, can be represented by a superposition of probabilities for air parcels reaching each grid area in the chosen domain or on a geographical map.

Let us consider several common approaches to construct probability fields based on trajectory modelling results (Baklanov and Mahura, 2001; Mahura, 2001; Mahura and Baklanov, 2002). For all approaches, initially, a gridded domain having $M_{\text {lat }} \times M_{\text {lon }}$ latitude vs. longitude grid points with a size of $\Delta Y \times \Delta X$ degrees latitude vs. longitude should be constructed. The selection of sizes $\Delta Y$ and $\Delta X$ depends on the resolution of original meteorological fields used for calculation of trajectories. The number of latitudinal and longitudinal grid points $-M_{l a t}$ and $M_{l o n}$ - is selected taking into account the farthest geographical boundaries which might be reached by air masses during the period studied. Time $t$ is the output modelling time interval which is equal $12 \mathrm{~h}$ for our study.

The first approach to construct such fields considers the number of trajectory intersections with each cell of the gridded domain $\left(N_{C E L L_{i j}}\right)$ :

$N_{C E L L_{i j}}=\sum_{k=1}^{N_{t r}} \sum_{j=1}^{M_{\text {lat }}} \sum_{i=1}^{M_{\text {lon }}} n_{i j k}$ $n_{i j k}=\left\{\begin{array}{l}0 \\ 1\end{array}\right.$ if $\quad\left\{\begin{array}{l}X_{i} \leq X_{k, t}<X_{i+1} \\ Y_{j} \leq Y_{k, t}<Y_{j+1}\end{array}\right.$,

where:

- $Y_{k, t}, Y_{k, t}$ - longitude and latitude of $k$-trajectory at time $t$

- $X_{i}, X_{i+1}$ - longitudinal boundaries of the grid cells of the gridded domain;

- $Y_{j}, Y_{j+1}$ - latitudinal boundaries of the grid cells of the gridded domain;

- $N_{t r}$ - total number of trajectories during the period studied (number of days considered $* 8$ trajectories per day);

- $M_{\text {lat }}, M_{\text {lon }}$ - number of the grid points in domain along latitude and longitude.

The second approach for construction of probabilistic fields uses an assumption that the total sum of contributions from all individual grid cells of domain is equal to $100 \%$. Hence, the contribution or probability that a given trajectory might reach the geographical boundaries of the individual cell could be estimated as follows:

$$
\begin{aligned}
& P_{i, j}=\frac{N_{C E L L_{i j}}}{N_{\text {tot }}} \cdot 100 \% \\
& N_{\text {tot }}=\sum_{i=1}^{M_{\text {lat }}} \sum_{j=1}^{M_{\text {lon }}} N_{C E L L_{i, j}},
\end{aligned}
$$

where:

- $P_{i, j}$ - probability of trajectory intersections with a particular cell of the gridded domain;

- $N_{t o t}$ - total number of trajectory intersections with all cells of the gridded domain.

The third approach for construction of probabilistic fields uses an assumption that for an individual site there is always a region where there is the highest probability of the maximum possible impact due to atmospheric transport. The detailed description of this approach is given in Sect. 2.4.1. In our study, the third approach to construct the probabilistic fields was selected as the most representative to evaluate the site possible impact compared with the previous two.

It should be noted that the most interest for further analysis would be the following indicators, based on the probabilistic fields: 1) airflow and fast transport, 2) maximum possible impact zone and maximum reaching distance, 3) typical transport time, and 4) precipitation factor. 


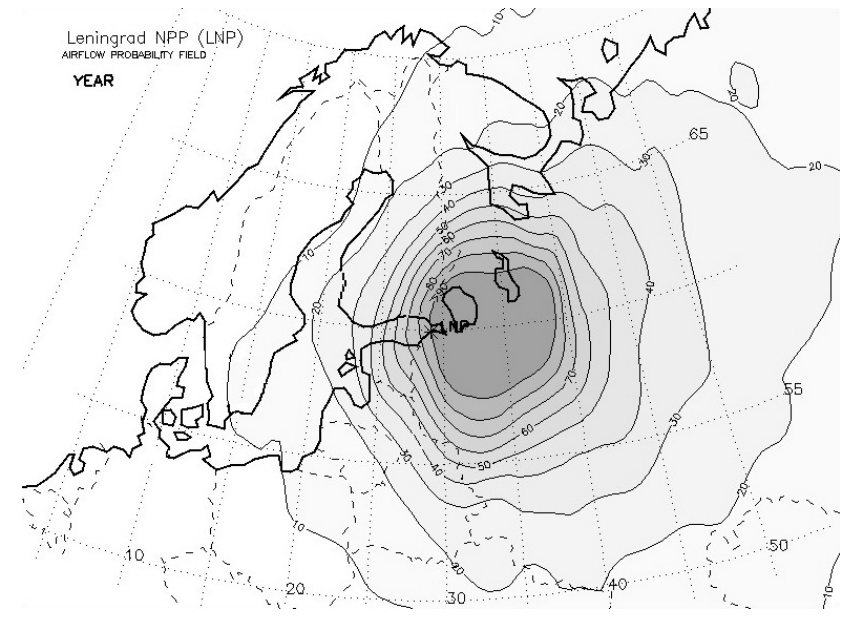

Fig. 2. Annual airflow probability field from the Leningrad nuclear power plant (isolines are shown in \% with respect to the area of the highest probability of possible impact).

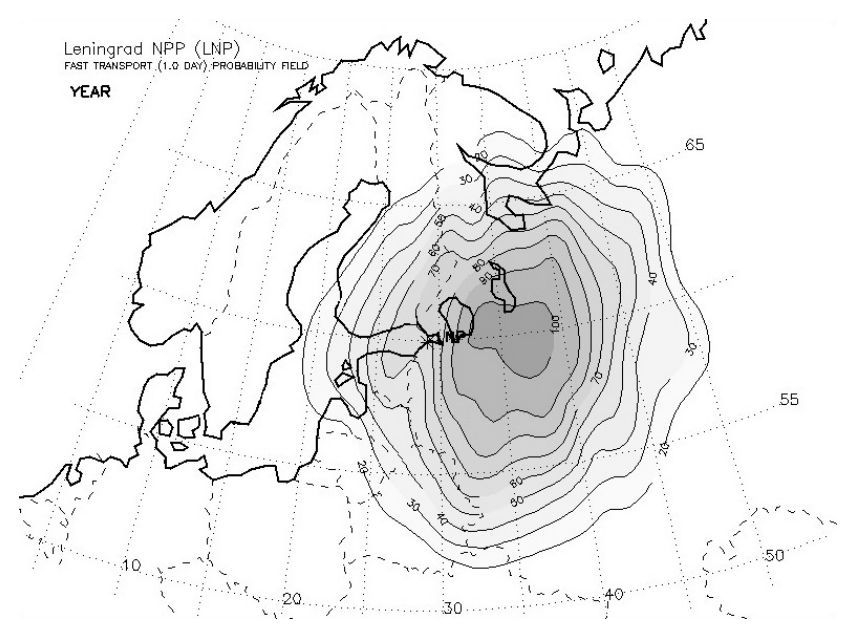

Fig. 3. Annual fast transport probability field after $24 \mathrm{~h}$ of atmospheric transport from the Leningrad nuclear power plant (isolines are shown in \% with respect to the area of the highest probability of possible impact).

\subsection{Indicators of the nuclear risk site possible impact}

\subsubsection{Airflow and fast transport probability fields}

The first type of probabilistic fields (airflow probability field, Fig. 2 shows the common features in the atmospheric transport patterns, i.e. it may provide a general insight on the possible main direction of the radioactive cloud transport as well as the possibility to reach or pass over any geographical area. The result of this analysis is an appropriate test to support or disprove results of the cluster analysis. This is because the atmospheric transport pathways (or mean trajectory clusters) show only the common direction of airflow away from the site. However, information between these pathways (or clusters) is missing.

The second type of probabilistic fields (fast transport probability field, Fig. 3 indicates the probability of the air parcels movement during the first day of transport. It is important information, especially, for estimating the short-living radionuclides - such as iodine isotopes - impact. These fast transport fields show those territories that may be reached after the first day, and those areas that are at the most danger due to fast transport probability.

These two types of probability fields are constructed using the third approach (see Sect. 2.3.2) based on assumption that for an individual site there is always a region where possible impact due to atmospheric transport might be the highest. The borders of such region could be estimated by comparing the number of trajectory intersections in the cells with the cell where the maximum number of intersections occurred: $\quad N_{A M C}=\max \left\{N_{C E L L_{1,1}}, \ldots, N_{C E L L_{M_{l a t}}, M_{l o n}}\right\}$. Hence, among all grid cells, the cell where the absolute maximum of intersections occurred would be identified as an "absolute maximum cell" (AMC). Because all trajectories start near the site region, to account for the contribution into the flow at larger distances from the site, the number of intersections in cells adjacent to AMC was compared, and then assigned additional cells, which had difference of less than $10 \%$ between cells. Therefore, this new "area of maxima", if isolines are drawn, will represent the area of the highest probability of the possible impact (AHPPI) from the site. Assuming a value of $100 \%$ for this area, the rest could be re-calculated as percentage of the area at the highest probability of the possible impact, or:

$$
\begin{aligned}
& P_{A H P M I_{i, j}}=\frac{N_{C E L L_{i j}}}{N_{D}} \cdot 100 \%, \\
& N_{D}=N_{t o t}-N_{A H P M I}, \\
& N_{A H P M I}=\sum_{j=1}^{M_{\text {lat }}} \sum_{i=1}^{M_{\text {lon }}} n_{i j},
\end{aligned}
$$

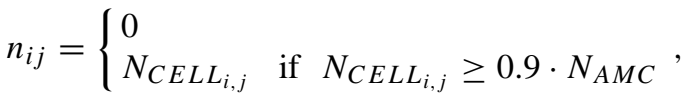

where:

- $P_{A H P M I_{i, j}}$ - probability of the NRS impact with respect to the area of the highest probability of the possible impact (AHPPI) of the nuclear risk site;

- $N_{D}$ - total sum of trajectory intersections with cells from the gridded domain, except the cells located in the boundaries of AHPPI for the nuclear risk site;

- $N_{A H P M I}$ - total sum of trajectory intersections with cells from the gridded domain located within the boundaries of AHPPI for the nuclear risk site. 
For example, as shown in Fig. 2, the isolines start from " $10 "(\%)$ and show contribution of cells into the total redistribution of the airflow around the site with respect to AHPPI. For this case, the boundaries of AHPPI (extended more in east-south sector from the site) are outlined by the isoline of " $>90 "(\%)$. This field also shows dominance of the westerly flows from the NRS region.

2.4.2 Maximum possible impact zone and maximum reaching distance

The third type of probabilistic fields, first, indicates boundaries of regions with the highest probability of being reached by trajectories during the first day of atmospheric transport from the risk sites. Let's call this indicator the maximum possible impact zone (MPIZ). Second, this type of the field also indicates the farthest boundaries on the geographical map that might be reached during the first day by, at least, one trajectory originating over the NRS location. Let's call it the maximum reaching distance (MRD).

To visualize the MPIZ indicator, all endpoints of calculated trajectories originated within the boundary layer were also counted (such calculation can be done for any other layer or altitude) during the first day of transport (at 12 and $24 \mathrm{~h}$ ). Then, a similar approach for construction of probability fields (as was used for the airflow and fast transport probability fields; see Sect. 2.4.1) was used to construct the MPIZ field. An isoline of MPIZ was drawn through the areas with the highest occurrence of trajectory intersections with the cells of the gridded domain (Fig. 4).

To visualize the MRD indicator, all endpoints of calculated trajectories at the end of the first day of atmospheric transport were used. An isoline of MRD was drawn through the grid cells where, at least, one trajectory intersected with the grid cell boundaries (Fig. 4). It should be noted also, that although the likelihood that an air parcel will reach these boundaries is low, it is still a possible case of atmospheric transport from the site.

\subsubsection{Typical transport time fields}

In the emergency response systems for nuclear accidents, the estimation of the radionuclide transport time to a particular territory, region, county, city, etc. is one of the important input parameters in the decision-making process. This information is extracted from the calculated isentropic trajectories and constructed the fourth type of probabilistic fields called the typical transport time (TTT) fields. These fields show: first, how long, typically, it will take for an air parcel to reach a particular geographical region from the NRS location, and second, what territories would be at the highest risk during the first few days of radionuclide cloud transport after an accident at NRS.

To construct the TTT fields, at the first step, a new polar grid domain having 36 sectors $\left(10^{\circ}\right.$ each) and 35 grid cells

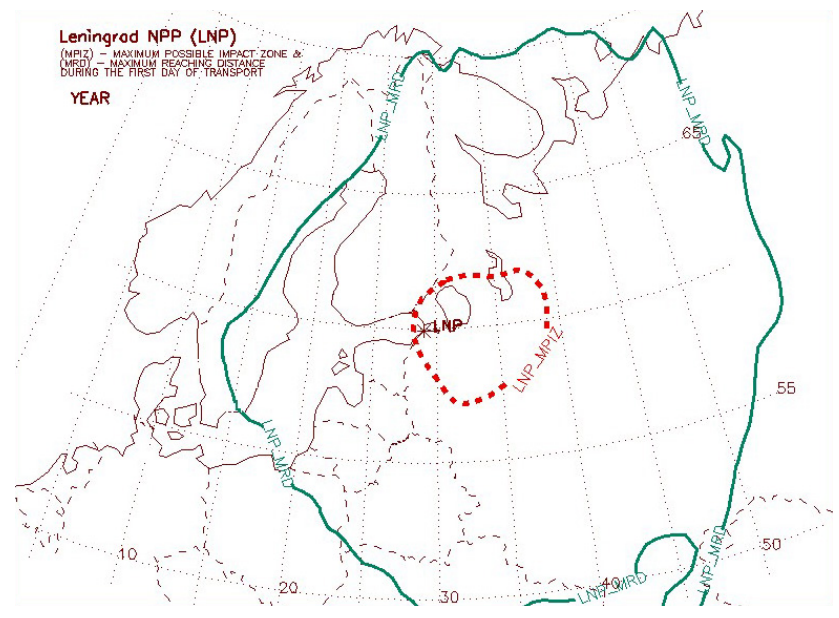

Fig. 4. Annual boundaries of the maximum reaching distance (-LNP_MRD-) and maximum possible impact zone (-LNP_MPIZ-) indicators for the Leningrad nuclear power plant.

$\left(2^{\circ}\right.$ each) along each sector line with the risk site in the center was built (although any other resolution of a grid domain could be chosen). At the second step, in the same way as in the probability fields analysis, the number of trajectory intersections was counted in each grid cell of new domain. Then, along each sector a grid cell with absolute maximum of trajectory intersections was selected, and an isoline of typical transport time was constructed.

A similar procedure is repeated for each selected temporal term. As a step of this procedure, initially, the geographical coordinates (latitude and longitude) were converted into polar coordinates (radius $R\left(X_{t}, Y_{t}\right)$ and polar angle $\alpha\left(X_{t}, Y_{t}\right)$ ):

$$
\begin{aligned}
& R\left(X_{t}, Y_{t}\right)=\sqrt{\left(X_{t}-X_{N R S}\right)^{2}+\left(Y_{t}-Y_{N R S}\right)^{2},} \\
& \gamma\left(X_{t}, Y_{t}\right)=\arctan \left(\frac{\left(Y_{t}-Y_{N R S}\right)}{\left(X_{t}-X_{N R S}\right)}\right), \\
& \alpha\left(X_{t}, Y_{t}\right)= \begin{cases}90-\gamma\left(X_{t}, Y_{t}\right) & \text { if } N-E \\
90+\gamma\left(X_{t}, Y_{t}\right) & \text { if } E-S \\
180+\gamma\left(X_{t}, Y_{t}\right) & \text { if } S-W \\
270+\gamma\left(X_{t}, Y_{t}\right) & \text { if } W-N\end{cases}
\end{aligned}
$$

where:

- $X_{t}, Y_{t}$ - longitude and latitude of trajectory at time $t$,

- $Y_{t}, Y_{N R S}$ - longitude and latitude of the NRS location,

- $\gamma\left(X_{t}, Y_{t}\right)$ - angle calculated for one of quadrants.

Then, for each time $t$ in each cell of the sector, a number of trajectory intersections $N_{C E L L_{i j}}(t)$ was counted, and compared with the cells along the sector line to find a cell with the absolute maximum of trajectory intersections $N_{A M C}(t)$ :

$N_{C E L L_{i j}}(t)=\sum_{j=1}^{M_{\text {sec }}} \sum_{i=1}^{M_{i n t}} n_{i j}$, 


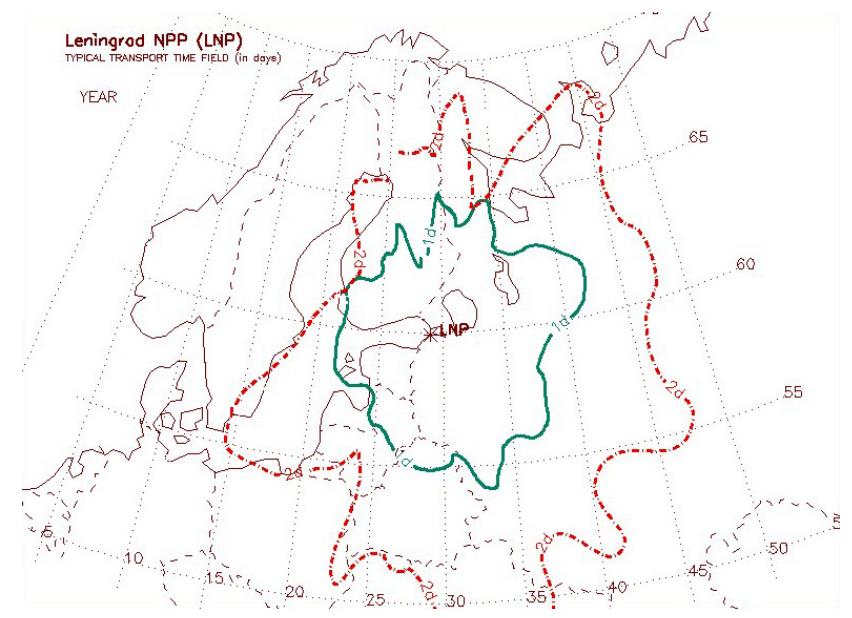

Fig. 5. Annual typical transport time fields at $1(-1 d-)$ and $2(-2 d-)$ days of atmospheric transport from the Leningrad nuclear power plant.

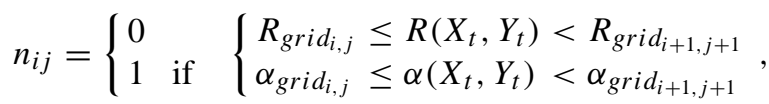

where:

- $R_{\text {grid }}, \alpha_{\text {grid }}$ - radius and angle of grid points in the gridded domain;

- $M_{\text {sect }}, M_{\text {int }}$ - total number of grid points of the gridded domain ( 36 sectors $\times 10^{\circ}$ and $35+1+35=71$ intervals $\times 2^{\circ}$, respectively).

For simplicity, if several AMC cells were identified along the sector line then the closest to the site was selected for construction of the TTT field (example of one- and two-day TTT is shown for the Leningrad NPP in Fig. 5. It should be noted that the TTT fields' construction for the terms later than 2.53 days is complicated due to significant airflow propagation from the site locations, and hence, the later terms isolines are not concentrated around the site and less representative.

\subsubsection{Removal or precipitation factor}

The wet deposition is an issue of most concern during atmospheric transport of radionuclide cloud over the geographical territories. It is highly temporally and spatially dependent. It plays important role in the estimation of the radionuclide surface deposition. To analyze the possible contribution of the removal processes during atmospheric transport from the NRS locations we might apply at least three different approaches.

The first approach is based on the evaluation of the precipitation climatology for the particular geographical area. Such climatological maps (on a multiyear and seasonal basis for the large scale domains) might be obtained from the meteorological weather services. These maps would reflect the accumulated precipitation measured near the surface for

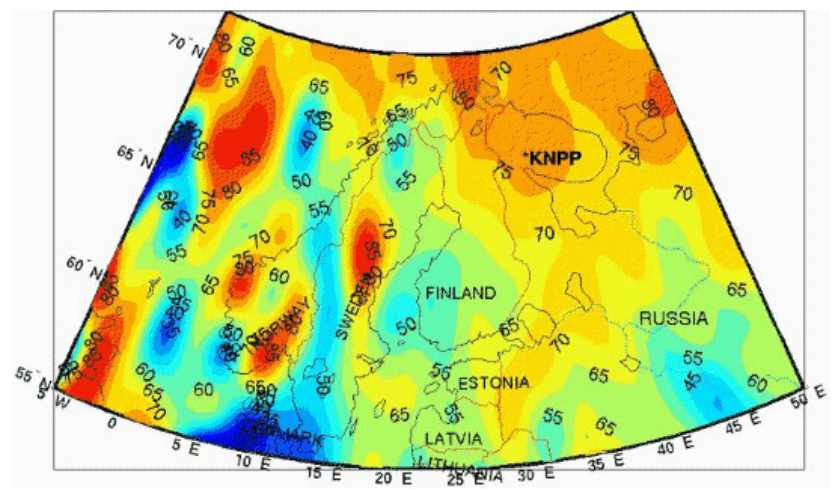

Fig. 6. Spring relative humidity (values are given in \%) or "precipitation factor" fields within the boundary layer for the Kola nuclear power plant.

each interval of time. It may be used for identification of the large size areas having common precipitation patterns. In particular, on such maps these areas are connected with the major centers of synoptic activity. However, air parcels might travel within different atmospheric layers during their transport from the NRS region. For example, if an air parcel travels in the free troposphere and there is no precipitation in this layer, but the area is marked as precipitable at the climatological map that will raise a misleading concern.

Therefore, the second approach is based on the evaluation of the probabilistic fields for the "precipitation factor" (ÖCB, 2000; INTAS, 2000; Mahura et al., 2001). An example is shown in Fig. 6. Relative humidity "plays a role" of the precipitation factor. At each selected time interval for each forward trajectory additional parameters including relative humidity can be calculated. It is one of the factors, which will determine the possibility of radionuclide removal during transport. Increasing relative humidity in the atmosphere is one of the signals of the water vapor increasing presence, and it may, in the presence of the cloud condensation nuclei, lead to formation of cloud cover. After clouds develop and form, under certain conditions there is a possibility of precipitation, and hence, radionuclide removal. Construction of the relative humidity fields is similar to the first steps in the probability field analysis. In this case, an average value of the relative humidity in each grid cell was calculated. Both the precipitation and relative humidity fields have a cellular figure in comparison with the airflow pattern. A pitfall in this analysis is the fact that all relative humidity values are directly related to the existing flow pattern. So, each field is valid only with respect to a particular risk site. Nevertheless, it is a more realistic pattern of the possible removal during transport than calculating rainfall climatological maps used in the first approach, because it includes processes above the surface. The limitation always is how we might resolve precipitation processes during air parcels transport. To resolve them we would need a finer meteorological data resolution. 
The third approach is based on the direct evaluation of the wet deposition fields at the ground surface (Baklanov et al., $2002 \mathrm{~b}$ ). It is also required to have multiyear output fields for comparison. For these purposes, we might run a dispersion and deposition model for a long-term period. Although this way is more accurate, it is more expensive computationally.

\section{Conclusions}

The methodology for assessment of nuclear risk and vulnerability for population in different geographical regions and countries in case of a severe accident at nuclear risk sites (NRSs) was outlined and discussed. The social-geophysical factors and indicators of the NRS possible impact which depend on the location of the area of interest and its population, were considered

The evaluation of atmospheric transport is given from the probabilistic point of view. In bounds of the probabilistic atmospheric studies several research tools were recommended to apply: (i) long-term trajectory modelling, (ii) a set of statistical methods to analyze trajectory modelling results, and (iii) constructing and mapping probabilistic fields of different NRS possible impact indicators due to atmospheric transport. Several useful indicators and methods of their construction were suggested and given. The indicators, based on results of trajectory modelling, are the following:

- atmospheric transport pathways,

- airflow probability fields,

- fast transport probability fields,

- typical transport time fields,

- maximum reaching distance,

- maximum possible impact zone,

- removal or precipitation factor.

It is assumed that these indicators will be applicable for further GIS-based analysis with integration of mathematical modelling to estimate risk and vulnerability as well as for the planning of emergency response and preparedness systems in cases of accidental releases at NRSs. The applicability of the methodology includes also:

- initial preliminary estimates of the atmospheric transport probability in event of an accidental release at NRS;

- improvement of systems for monitoring and emergency response to accidental releases;

- input for the social and economical studies of consequences due to the NRS impact on population and environment of the neighbouring territories and countries;
- input for the multidisciplinary risk and vulnerability analyses, probabilistic assessment of radionuclide local-, regional-, and long-range transport;

- modelling and testing of the higher resolution models.

Acknowledgements. The authors are grateful to L. Laursen and J. H. Sørensen (Danish Meteorological Institute), O. Rigina (Technical University of Denmark), R. Bergman (Swedish Defence Research Authority), B. Segerståhl (Thule Institute, University of Oulu, Finland), J. Merrill (University of Rhode Island, USA), F. Parker (Vanderbilt University, USA), K. Compton (International Institute for Applied Systems Analysis, Austria), S. Nielsen (Ris $\varnothing \mathrm{Na}-$ tional Laboratory, Denmark), and S. C. Hoe (Danish Emergency Management Agency) for collaboration, discussions and constructive comments. Thank you to $H$. van Dop and anonymous referee for constructive criticism and comments on the manuscript of this paper. Financial support of this study included the grants of the Nordic Arctic Research Programme and Nordisk Forskerutdanningsakademi.

\section{References}

Andreev, I., Hittenberger, M., Hofer, P., Kromp-Kolb, H., Kromp, W., Seibert, P., and Wotawa, G.: Risks due to severe accidents of nuclear power plants in Europe - the methodology of Riskmap, J. Hazardous Materials, 61, 257-262, 1998.

AR-NARP: On-going Project Atmospheric Transport Pathways, Vulnerability and Possible Accidental Consequences from the Nuclear Risk Sites in the European Arctic (Arctic Risk) of the NARP: Nordic Arctic Research Programme, WWW-site: http: //www.dmi.dk/f+u/luft/arctic-risk/main.html, 2001-2003.

Baklanov, A., Tausnev, N., Morozov, S., Nazarenko, L., Zolotkov, A., Bakulin, L., Barsukov, I., Rigina, O., Rodyushkina, I., Smagin, A., Klyuchnikova, E., Cherepanov, S., Mahura, A., and Limkina, S.: Determination of risk zones and elaboration of scenarios of extreme radiologically dangerous situations in the Northern areas, Report for the Russian Federation Ministry of Environment according to Theme "RISK" (\#2416) of the State Programme "Ecological Safety of Russia", Apatity: INEP KSC RAS, 179 p., 1992.

Baklanov, A., Mahura, A., and Morozov, S.: The Simulation of Radioactive Pollution of the Environment after a Hypothetical Accident at the Kola Nuclear Power Plant, Journal of Environmental Radioactivity, 25, 65-84, 1994.

Baklanov, A. and Bergman, R.: Radioactive Sources in the Barents Euro-Arctic Region: Are there reasons to be concerned?, Chapter XI at the NEBI Yearbook: North European and Baltic Sea Integration, Copenhagen, Springer-Verlag, 171-192, 1999.

Baklanov, A., Bergman, R., Lundström, C., and Thaning, L.: Modeling of episodes of atmospheric transport and deposition from hypothetical nuclear accidents on the Kola Peninsula, CERUM Northern Studies, No. 23., Umeå University, Sweden, 2001.

Baklanov, A. and Mahura, A.: Atmospheric Transport Pathways, Vulnerability and Possible Accidental Consequences from $\mathrm{Nu}-$ clear Risk Studies: Methodology for Probabilistic Atmospheric Studies, Danish Meteorological Institute Scientific Report, 0109, ISBN:87-7478-450-1, 43 p., 2001. 
Baklanov, A., and Sørensen, J. H.: Parameterisation of radionuclide deposition in atmospheric dispersion models, Phys. Chem. Earth, (B), 26, 787-799, 2001.

Baklanov, A., Mahura, A., Jaffe, D., Thaning, L., Bergman, R., and Andres, R.: Atmospheric Transport Patterns and Possible Consequences for the European North after a Nuclear Accident, Journal of Environment Radioactivity, 60, 1-26, 2002a.

Baklanov, A., Mahura, A., Sørensen, J. H., Rigina, O., and Bergman, R.: Methodology for Risk Analysis based on Atmospheric Dispersion Modelling from Nuclear Risk Sites, NARP Arctic Risk project, Danish Meteorological Institute Scientific Report, 02-16, ISBN:87-7478-470-6, 54 p., 2002 b.

Baklanov, A.: Methodologies for multidisciplinary nuclear risk and vulnerability assessments in the Arctic and Sub-Arctic, NATO Science Series, Kluwer Academic Publishers, Ser. IV, Earth and Env. Sci., Vol. 31, 385-405, 2003.

Baklanov, A., Mahura, A., and Sørensen, J. H.: Methodology for Prediction and Estimation of Consequences of Possible Atmospheric Releases of Hazardous Matter: Kursk Submarine Study, Atmospheric Chemistry and Physics, 3, 747-762, 2003.

Bergman, R. (Ed.): Assessment of potential risk of environmental radioactive contamination in northern Europe from terrestrial nuclear units in north-west Russia, INTAS project 96-1802I, Technical report \# 1 for February 1998-February 1999, FOA-R-9901080-861-SE, 109 p., 1999.

Bergman, R. and Baklanov, A.: Radioactive sources in main radiological concern in the Kola-Barents region, FRN-FOA publication, Stockholm, July 1998, 82 p., 1998.

Bergman, R., Thaning, L., and Baklanov, A.: Site-sensitive hazards of potential airborne radioactive release from sources on the Kola Peninsula, FOA report: FOA-R-00717-861-SE, February 1998, 14 p., 1998.

Bergman, R. and Ågren, G.: Radioecological Characteristics of Boreal or Sub-Arctic Environments in Northern Sweden: focus on long-term transfer of radioactive deposition over food-chains, The 4th International conference on Environmental Radioactivity in the Arctic, Edinburgh, Scotland, 20-23 September 1999, 91-94, 1999.

Dahlgaard, H. (Ed.): Nordic Radioecology: The transfer of radionuclides through Nordic ecosystems to man, Amsterdam, Elsevier Science B. V., Studies in Environmental Science, No 62, 483 p., 1994.

Eliassen, A. and Saltbones, J.: Modelling of long-range transport of sulphur over Europe: A two-year model run and some model experiments, Atmospheric Environment, 17, 8, 1457-1473, 1983.

Galmarini, S., Graziani, G., and Tassone, C.: The atmospheric long range transport model LORAN and its application to Chernobyl release, Environmental Software, 7, 3, 143-154, 1992.

Galmarini, S., Bianconi, R., Bellasio, R., and Graziani, G.: Forecasting the consequences of accidental releases of radionuclides in the atmosphere from ensemble dispersion modelling, Journal of Environmental Radioactivity, 57, 3, 203-219, 2001.

IIASA: Baklanov, A., Bergman, R., and Segerståhl, B.: Radioactive sources in the Kola region: Actual and potential radiological consequences for man, Report, International Institute for Applied Systems Analysis, Laxenburg, Austria, IIASA, Radiation Safety of the Biosphere, 255 p., 1996.

INTAS: Assessment of Potential Risk of Environmental Radioactive Contamination in Northern Europe from Terrestrial $\mathrm{Nu}$ - clear Units in North-West Russia, Final Research Report, INTAS Project 96-1802, November 2000, Apatity-Umeå, 125 p., 2000.

Jaffe, D., Mahura, A., and Andres, R.: Atmospheric Transport Pathways to Alaska from Potential Radionuclide Sites in the Former Soviet Union, Research Report, UAF-ADEC Project 96-001, 71 p., 1997a.

Jaffe, D., Mahura, A., Andres, R., Baklanov, A., Thaning, L., Bergman, R., and Morozov, S.: Atmospheric Transport from the Kola Nuclear Power Plant, Research Report, UAF-FOA-BECN Joint Project, BECN: Tromsø University, Norway, Fall 1997, 61 p., 1997b.

Kahl, J. D.: On the prediction of trajectory model error, Atmospheric Environment, 30, 2945-2 957, 1996.

Mahura, A. G.: Atmospheric transport pathways to Alaska from the Bilibino nuclear power plant, MS Thesis, University of Alaska Fairbanks, 87 p., 1998.

Mahura, A. G., Jaffe, D., Andres, R., and Merrill, J.: Atmospheric transport pathways from the Bilibino nuclear power plant to Alaska, Atmospheric Environment, 33/30, 5115-5122, 1999.

Mahura, A., Andres, R., and Jaffe, D.: Atmospheric transport patterns from the Kola Nuclear Reactors, CERUM Northern Studies No. 24, Umeå University, Sweden, 33 p., 2001.

Mahura, A.: Probabilistic Assessment of Atmospheric Transport Patterns from Nuclear Risk Sites, Ph.D. Thesis, Russian State Hydrometeorological University/ Kola Science Center, 172 p., 2001.

Mahura, A. and Baklanov, A.: Probabilistic Analysis of Atmospheric Transport Patterns from Nuclear Risk Sites in EuroArctic Region, Danish Meteorological Institute Scientific Report, 02-15, ISBN: 87-7478-469-2, 87 p., 2002.

Miller, J. M.: A Five-Year Climatology of Back Trajectories from Mauna-Loa Observatory, Hawaii, Atmospheric Environment, 15, 1553-1558, 1981.

Merrill, J., Bleck, R., and Boudra, D. B.: Techniques of Lagrangian Trajectory Analysis in Isentropic Coordinates, Monthly Weather Review, 114, 571-581, 1985.

Moberg, L. (Ed.): The Chernobyl fallout in Sweden, Results from a research programme on environmental radiology, The Swedish Radiation Protection Institute, Stockholm, Sweden, 1991.

Nielsen, S. P.: A sensitivity analysis of a radiological assessment model for Arctic waters, Radiation Protection Dosimetry, 75, 213-218, 1998.

ÖCB: Nuclear Risks, Environmental and Development Cooperation in the North of Europe, FRN publication, CERUM, University of Umeå, Sweden, 240 p., 2000.

Penenko, V., and Baklanov, A.: Methods of sensitivity theory and inverse modeling for estimation of source parameters and risk/vulnerability areas, Lecture Notes in Computer Science, LNCS, 2074, 57-66, 2001.

Rigina, O.: Integration of Remote Sensing, mathematical modelling and GIS for complex environmental impact assessment in the Kola Peninsula, Russian North, Ph.D. thesis, April 2001, Geographical Institute of Copenhagen University, Copenhagen, IGUK press, 2001.

Rigina, O. and Baklanov, A.: Regional radiation risk and vulnerability assessment by integration of mathematical modeling and GIS-analysis, J. Environment International, 27, 6, 1-14, 2002.

Saltbones, J., Foss, A., and Bartnicki, J.: Threat to Norway from potential accidents at the Kola nuclear power plant, Climatolog- 
ical trajectory analysis and episode studies, Atmospheric Environment, 34, (3), 407-418, 2000.

Sass, B. H., Nielsen, N. W., Jørgensen, J. U., Amstrup, B., and Kmit, M.: The operational HIRLAM system, DMI Technical report 00-26, Copenhagen, Denmark, 2000.

Slaper, H., Eggink, G. J., and Blaauboer, R. O.: Risk assessment method for accidental releases from nuclear power plants in $\mathrm{Eu}-$ rope, Report of the National Institute of public health and the environment, Bilthoven, The Netherlands, 1994.
Stohl, A.: Computation, accuracy and applications of trajectories - A review and bibliography, Atmospheric Environment, 32, 6, 947-966, 1998.

Sørensen, J. H.: Sensitivity of the DERMA Long-Range Gaussian Dispersion Model to Meteorological Input and Diffusion Parameters, Atmos. Environ. 32, 4195-4206, 1998.

Sørensen, J. H., Laursen, L., and Rasmussen, A.: Use of DMIHIRLAM for operational dispersion calculations, Air Pollution Modeling and Its Application X., 1994. 